\title{
A Review - Signature Verification System Using Deep Learning: A Challenging Problem
}

\author{
Punam R. Patil ${ }^{11}$, Bhushan V. Patil ${ }^{2}$ \\ ${ }^{*}$ Computer Engineering, R. C. Patel Institute of Technology,Shirpur, Maharashtra, India \\ 2Electronics and Telecommunication Engineering, R. C. Patel Institute of Technology, Shirpur, Maharashtra, \\ India
}

\begin{abstract}
Article Info

Volume 8 Issue 2

Page Number: 295-298

Publication Issue :

March-April-2021

Article History

Accepted : 11 April 2021

Published : 17 April 2021

One of the challenging and effective way of identifying person through biometric techniques is Signature verification as compared to traditional handcrafted system, where a forger has access and also attempt to imitate it which is used in commercial scenarios, like bank check payment, business organizations, educational institutions, government sectors, health care industry etc. so the signature verification process is used for human examination of a single known sample. There are mainly two types of signature verification: static and dynamic. i) Static or off-line verification is the process of verifying an electronic or document signature after it has been made, ii) Dynamic or on-line verification takes place as a person creates his/her signature on a digital tablet or a similar device. As compared, Offline signature verification is not efficient and slow for a large number of documents. Therefore although vast and extensive research on signature verification there is need to more focus and review on the online signature verification method to increase efficiency using deep learning.
\end{abstract}

Keywords : Signature Verification, Static, Dynamic, Deep Learning.

\section{INTRODUCTION}

In last few years it has been seen that personal identity as an authentication is one of the growing interest. Therefore, authentication consider as a deepdown part in social life. Higher security in increasing demand put much more attention on biometrics (i.e individual recognition based on personal characteristics) for verifying a person. Generally, among distinct biometric parameters for
Identification of a person on any document drawn through a uniquely outlined writes as an identity by using a signature. Any individual on daily basis uses it for any legal documents whenever required. A signature through any person convey a picture of particular pattern of pixels. Therefore one of the most common and effective way to identify person through signature is handwritten signature identification. From different reviews of distinct proposed systems clarifies more focus on verification than that of 
identification because of usage of signature in daily life usage [6]. So the serious matter arises with this system when anyone trying to replicate that individual's signature for any purposes.

There are mainly two types of verification of signature, i) Offline/Static verification

Online/Dynamic verification. Static verification system captures shape of signature so input data includes $\mathrm{x}, \mathrm{y}$ coordinates of signature. The dynamic verification system uses devices for capturing surplus information like pen up and down, time, pressure, azimuth etc. Previously researches listing and describing the below approaches for signature verification are listed in table 1 as [6],

Table 1: Approaches of signature verification

\begin{tabular}{|c|c|c|c|}
\hline $\begin{array}{l}\text { Sr. } \\
\text { No. }\end{array}$ & $\begin{array}{c}\text { Verification } \\
\text { approach }\end{array}$ & Discussion & $\begin{array}{l}\text { Techniques } \\
\text { prefer }\end{array}$ \\
\hline 1 & $\begin{array}{l}\text { Template } \\
\text { Matching }\end{array}$ & $\begin{array}{l}\text { Templates } \\
\text { signature } \\
\text { against a } \\
\text { questioned } \\
\text { sample }\end{array}$ & $\begin{array}{l}\text { Euclidian } \\
\text { distance } \\
\text { DTW- } \\
\text { Dynamic } \\
\text { Time } \\
\text { Wrapping }\end{array}$ \\
\hline 2 & Statistical & $\begin{array}{l}\text { Distance- } \\
\text { based } \\
\text { classifiers } \\
\text { are used. }\end{array}$ & $\begin{array}{l}\text { Neural } \\
\text { Networks } \\
\text { HMM- } \\
\text { Hidden } \\
\text { Markov } \\
\text { Model }\end{array}$ \\
\hline 3 & Structural & $\begin{array}{l}\text { Structural } \\
\text { representatio } \\
\text { ns }\end{array}$ & $\begin{array}{l}\text { Graph/tree } \\
\text { matching } \\
\text { techniques }\end{array}$ \\
\hline
\end{tabular}

Till today most of the work done related to deep learning for various biometrics like fingerprint, face, iris and finger vein. So, in this paper most of the attention on to review of proposed system that using deep learning technique for signature verification purpose.

B. Significance of the study: This study will help us to,

i) How to obtain a fixed-sized vector representation for signatures of varied size,

ii) How the resolution of the scanned signatures impact system performance

iii) The impact of fine-tuning representations to other operating conditions (e.g. different acquisition protocols, signatures from people of different locations), by using transfer learning to other datasets.

iv) Low number of samples per user for training

v) Presence of partial knowledge during training

\section{LITERATURE REVIEW}

One of the important behavioural biometrics is handwritten signing, which are usually accepted for verification purposes like legal documents authentication, financial contracts purpose etc. In this paper author focus on to model the signatures using Legendre polynomials coefficients as one of the feature. Also, as a classifier deep feed forward neural network and DLL is stochastic gradient descent with momentum. Author performs experimentation on SigComp2011 Dataset which concludes better equal error rate reduction and enhancement in accuracy as compared to state-of- the-art methods [2].

In this paper author focus on one of the accurate method used for signature verification where more attention on difficulties like lacking of efficient samples for training, methods must be spatial change invariant etc. Hence author proposed a method that each user with a one-class classifier is built on discriminative features. Firstly, using a large number of unlabelled signatures, pre-train a sparse autoencoder then on this applied the discriminative features, which are learned by auto- encoder to 
represent the training and testing signatures as a selfthought learning method. Finally through a one-class classifier, user's signatures are modelled and classified. This proposed method is independent on signature datasets, though experimental results clarifies significant error reduction and accuracy enhancement on SVC2004 and SUSIG datasets [6].

Signature, one of the precarious element for authentication and authorization in commercial, financial and legal transactions which is decently one of the most frequently used biometrics for authentication. Therefore an accurate and efficient signature verification system is more preferable. One of the challenging task of this system is to discriminate the original signatures from the forged ones. The author in this paper focus on more challenging one offline signature system although varied research done in last decade, but still it faces some problems yet. As signature verification system consist of three phases mainly as i) image preprocessing, ii) feature extraction, and iii) verification. Therefor author in this paper proposed and focus on two features like a) Stroke angle and average intersected points b) Pixel density of the signature nucleus, which in turn leads to strengthen of set of features with proposed features to get verification of signature accurately [7].

One common task in forensic document analysis is Signature verification for determination of signature matches with given sample. From the view of automating the task that involves machine learning (ML) from a population of signatures. In this paper author proposed two kinds of learning,

i) set of genuine and forgeries from a population (person-independent or general learning, ii) there are genuine signatures person-dependent or special learning. In general learning- from a population the differences in between genuine and forgeries across all individuals are learnt and in special learning, a person's signature is learnt from multiple samples of only that person's signature- within-person similarities are learnt. In special learning, a person's signature is learnt from multiple samples of only that person's signature but general learning model allows a questioned signature to be compared to a single genuine signature. For a sufficient number of samples, special learning type performs better than general learning [8].

Authentication plays an important role in regards to manage security. With advancement in technology, to interact with machine made possible, that leads to increasing need of authentication for security purpose. So today more attention on biometric-based authentication. In this paper author proposed a method for pre-processing of signatures to make verification simple through a novel method of signature recognition and signature forgery detection with verification using Convolution Neural Network (CNN), Crest-Trough method and SURF algorithm \& Harris corner detection algorithm [9].

\section{CONCLUSION}

As we know that Signature verification system works in static as well as in dynamic nature. But to overcome the disadvantages of static system in this paper we focused on as compared to existing technologies much more attention and need of a new proposed approach that has been introduced to verify the online signatures depending upon self-thought learning. Which shows effective review on different proposed techniques. As a future work, this method can be tested on deep convolutional networks on both online and offline signature datasets. Therefore although vast and extensive research on signature verification there is need to more focus and review on the online signature verification method to increase efficiency using deep learning.

Conflicts of Interest: The authors declare no conflict of interest. 


\section{REFERENCES}

[1]. Hsin-Hsiung Kao and Che-Yen Wen, "An Offline Signature Verification and Forgery Detection Method Based on a Single Known Sample and an Explainable Deep Learning Approach", Appl. Sci. 2020, 10, 3716; doi:10.3390/app10113716.

[2]. Amr Hefny and Mohamed N Moustafa, "Online Signature Verification Using Deep Learning and Feature Representation Using Legendre Polynomial Coefficients", The International Conference on Advanced Machine Learning Technologies and Applications (AMLTA2019), DOI: 10.1007/978-3-030-14118-9_68, January 2020.

[3]. Eman Alajrami et.al., "Handwritten Signature Verification using Deep Learning", International Journal of Academic Multidisciplinary Research (IJAMR), Vol. 3 Issue 12, December - 2019, Pages: 39-44, ISSN: 2643-9670.

[4]. Gopichand G et.al., "Digital Signature Verification Using Artificial Neural Networks", International Journal of Recent Technology and Engineering (IJRTE), ISSN: 2277-3878, Volume-7 Issue-5S2, January 2019.

[5]. Debasree Mitra et.al., "Machine Learning Approach for Signature Recognition by HARRIS and SURF Features Detector", INTERNATIONAL JOURNAL OF COMPUTER SCIENCES AND ENGINEERING, DOI: 10.26438/ijcse/v7i5.7380, May 2019.

[6]. Mohammad Hajizadeh Saffar et.al., "Online Signature Verification using Deep Representation: A new Descriptor", 24 Jun 2018.

[7]. Md. Aminur Rahman et.al., "Writerindependent Offline Handwritten Signature Verification using Novel Feature Extraction Techniques", International Journal of Computer
Applications (0975 - 8887), Volume 177 - No. 14, October 2019.

[8]. Harish Srinivasan et. al., "Machine Learning for Signature Verification", ICVGIP 2006, LNCS 4338, pp. 761-775, 2006 (C) Springer-Verlag Berlin Heidelberg 2006.

[9]. Jivvesh poddar et.al., "Offline Signature Recognition and Forgery Detection using Deep Learning", The 3rd International Conference on Emerging Data and Industry 4.0 (EDI40), Procedia Computer Science 170 (2020) 610617, Warsaw, Poland, April 6 - 9, 2020.

\section{Cite this article as :}

Punam R. Patil, Bhushan V. Patil, "A Review Signature Verification System Using Deep Learning: A Challenging Problem", International Journal of Scientific Research in Science, Engineering and Technology (IJSRSET), Online ISSN : 2394-4099, Print ISSN : 2395-1990, Volume 8 Issue 2, pp. 295-298, March-April 2021. Available at doi : https://doi.org/10.32628/IJSRSET207632 Journal URL : https://ijsrset.com/IJSRSET207632 